\title{
Substantial Content Reclamation for Clustering
}

\section{Rajeev Tripathi}

\begin{abstract}
The massive volume of data stored in computer files and databases is rapidly increasing. Users of these data, on the other hand, demand more complex information from databases. The video data have exponential growth towards accessing and storing. The vital problem associated to video data is efficient, qualitative and fast accessing. We talk about how video pictures are clustered. We presume video clips have been divided into shots, each of which is denoted by a collection of key frames. As a result, video clustering is limited to still key frame pictures. In amble database finding the qualified data set (clusters) is quite time-taking job. The video data mining relate to multi-lingual text, numeric, image, video, audio, graphical, temporal, relational and categorical data. It may be any kind of information medium that can be represented, processed, stored, fast accessing or summarization of clusters are required due to which significant frame-set is formed. Due to sampling error and test reliability in video, substantial changes of more than one frame are predicted. The goal of this article is to show how to employ a familiar and easy nonparametric statistical approach (chi-square) to select eligible data/framesets for analysis. The chi-square model illustrated here is a straightforward, sensible, fast, reduce saddle, and easiest method. Skimming/ Summarization and clipping technique are further enhanced by this technique along with video database maintenance technique from simple descriptors to a complex description schemes like spatial and temporal or high dimensional indexing.

Keywords: Data mining, Clusters, Chi-square, NonParametric, Skimming, Text Mining
\end{abstract}

\section{INTRODUCTION}

Our ability to generate and gather data has been constantly increasing. Data is generated via the ubiquitous use of digital cameras, publications, and bar codes, in addition to the computerization of most corporate, scientific, and government interactions. Scannable text, picture platforms, satellite remote sensing technologies, and the World Wide Web have showered us with a massive quantity of data on the congregational side. The need for innovative approaches and automated technologies to help us turn this data into usable information and knowledge has grown even more essential as a result of this quick development.

It is impractical to treat a video clip as an enormous sequence of separate still photographs and examine each picture in video or multimedia since there are just too many pictures, and most adjacent images may be rather identical.

It is preferable to approach each video clip as a collection of action and events in time and then temporarily split them into video shots in order to capture the storyline or event structure of a film.

Manuscript received on August 08, 2021.

Revised Manuscript received on August 17, 2021.

Manuscript published on September 30, 2021.

* Correspondence Author

Dr. Rajeev Tripathi*, Assistant Professor, Department of Computer Application, Shri Ramswaroop Memorial Group of Professional Colleges Lucknow, India, Email: rajeev2363@gmail.com

(C) The Authors. Published by Blue Eyes Intelligence Engineering and Sciences Publication (BEIESP). This is an open access article under the CC BY-NC-ND license (http://creativecommons.org/licenses/by-nc-nd/4.0/)
A shot is a collection of frames or images in which the video material does not suddenly change from one frame to the next. Furthermore, the most illustrative frame in a video clip is assessed as the shot's key frame. In content-based image retrieval, each key frame may be analyzed utilizing image feature extraction and analysis methods. The key frame sequence will then be utilized to specify the order in which the events in the video clip will occur. As a result, in video processing and mining, the identification of shots and the extraction of crucial frames from video streams become critical jobs.

A technique for skimming digital audio and video data, where in the video data is sectioned into video segments and the audio data has been transcribed, is encompassed of the steps of choosing representative frames from each of the video segments. The illustrative frames are combined to form an assembled video sequence. Keywords delimited in the corresponding transcribed audio data are recognized and extracted. The extracted frames are assembled in to an audio track. The accumulated video sequence and audio track are output organized.

For such method of skimming [1,7-8] the key frame election and detection is a quite algorithmic Job. The statistical derivation is quite helpful for this processes/method. In the presented paper we suggest for nonparametric test for the election of the key frames over the video sequence.

Because the data does not have to fit into a normal distribution, nonparametric tests are also known as distribution free statistics. Nonparametric tests, in general, need less stringent data assumptions. Another benefit of utilizing these tests is that they can be used to examine both category and rank data.

Nonparametric tests use fewer assumptions and are applicable to a wider range of data types. The relationship between parametric tests and the statistical test conclusion should assist put nonparametric testing into situation with what we've studied. For instance, we have previously erudite about the binomial test for the simplest case of nominal data and Spearman's Rho for correlation concerning rank data for the purpose used the Chi- Square test. It is essential to note that still with metric data. If expectations are deficiently desecrated nonparametric tests are probable to be engaged.

\section{LITERATURE REVIEW}

\section{Chi - Square and independent samples:}

The chi-square(2)[2] test for nominal (categorical) data is one of the most commonly used statistical tests, and it has been used to a extensive variety of difficulties and troubles involving frequency data [5].

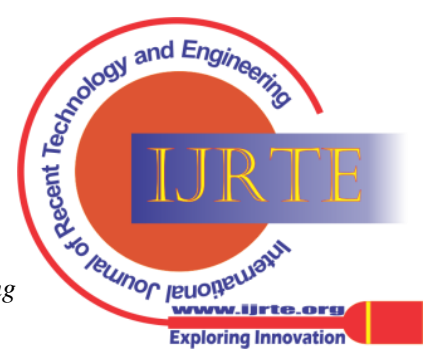


The content classifications must be autonomous and reciprocally restricted, which is the fundamental criteria of the 'chi-square' test. In that specific cluster [4] or data segment, the video data is notional data. The content groups are autonomous and reciprocally exclusive. So that cluster A is much diverse from cluster $B$.

\section{The $\left(\chi^{2}\right)$ formula:}

The following is an instance of how the chi-square statistic is utilized in frequency of video data analysis. We want to know if the observed and obtained frequencies for a video data set relating to a video - clip deviate significantly from the rates we expected to see. The chi-square test is used to make this decision. The chi-square value is calculated using the consequent regular equation.

$$
\chi^{2}=\sum \frac{\left.\left.\sum \mathrm{f}_{\mathrm{o}}-\mathrm{f}_{\mathrm{e}}\right)^{2}\right]}{\mathrm{fe}_{\mathrm{e}}}
$$

The $\left(\chi^{2}\right)$ value above is resultant from the sum of the experimental values minus the conventional values squared $\left(f_{0}-f_{e}\right)^{2}$ divided by the predictable value $\left(f_{e}\right)$. Clearly, if the actual values equivalent the predictable value, the (2) value is zero, signifying that there is no disparity between what we saw and what we expected to see based on the (2) distribution probabilities.

\section{Expected values:}

In the $\left(\chi^{2}\right)$ analysis, the predictable values can generally be consequent through one of three ways: through change (probability), through an a priori theory or hypothesis or through accessible data and research. The latter model, commonly referred to as an "empirical" model, is the one utilized to generate the predicted video data values in this case. Video data tends to fluctuate between average and actual due to differences in different standards.

To obtain the empirical expectancy approximation, one can average a large number of video data points; however, the numeral of points used to create the experimental anticipation approximations must be carefully considered, as averaging out a large number of data points can both rounded and disguise rapidly emerging "Actual" changes as opposed to large standard changes and major parameter shifts. However, the goal here is to detect statistically significant rate shifts in the categories of interest as they emerge quickly and reliably. By developing an empirical standard for analyzing and deciding on percentages objectively rather than subjectively.

One of the assumptions of $(\chi 2)$ is that no predictable category should be less than one. This is a significant point to consider when working with video data, because if we do not expect to see variance in video sequences by a specific video standard at all in observation, then the occurrence of variation to any degree is considerable not considering of sample size, matrix, or test statistic or level of implication used.

\section{PROPOSED METHODOLOGY}

\section{Sample Size:}

A suitably big sample is one of the key assumptions of $\left(\chi^{2}\right)$. When $\left(\chi^{2}\right)$ is applied to tiny samples, the probability of type II errors rises to intolerable levels [5]. With video data, sample size is seldom an issue because sample sizes are often big (more than 100), however clips that are encountered infrequently would clearly have lower testing frequencies. This criteria is appropriate to be relevant to the $(\chi 2)$ analysis of video data presented above, and data from the sample seven frame set findings may be corrected using corrective correction techniques.

\section{Goodness of fit tests for a one categorical variable:}

A. We is interested in determining chosen frame frequency from given video frame-set are significantly acceptable or not.

B. The 5-step approach to hypothesis testing

1. $\mathrm{H}_{0}$ : Numbers of Frames are acceptable(or suitable)

$\mathrm{H}_{1}$ : Numbers of frames are not acceptable

2. The implication level is 05 .

3. Chi- square is the examination statistic.

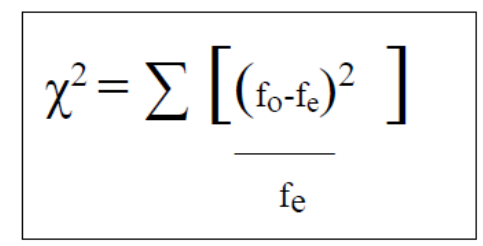

Figure 1. $\chi^{2}$ Formula

4. The decision rule:

If $\chi^{2}$ from the test statistic is exterior the critical value, the variance is high and the null hypothesis is rejected.

5. Put on the decision rule for given one tail test.

$\mathrm{df}=\mathrm{k}-1=7-1=6 \rightarrow \chi^{2}=12.592$

Select $\mathrm{H}_{0}$ because $12.05<12.592$.

Numbers of Frames are acceptable

Figure 2. $\chi^{2}$ Calculated Value

\section{RESULT ANALYSIS}

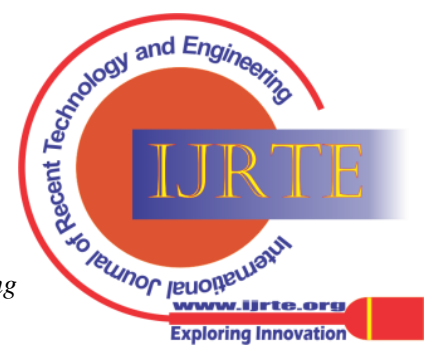


Table 1. Input data

\begin{tabular}{|c|c|c|c|c|c|c|c|c|}
\hline Frame & $\mathrm{A}$ & $\mathrm{B}$ & $\mathrm{C}$ & $\mathrm{D}$ & $\mathrm{E}$ & $\mathrm{F}$ & $\mathrm{G}$ & Total \\
\hline $\begin{array}{c}\text { Occurrence } \\
\text { Observed } \\
\mathrm{f}_{\mathrm{o}}\end{array}$ & 12 & 16 & 15 & 9 & 16 & 9 & 3 & 80 \\
\hline $\begin{array}{c}\text { ccurrence } \\
\text { Expected } \mathrm{f}_{\mathrm{e}}\end{array}$ & 11.428571 & 11.428571 & 11.428571 & 11.428571 & 11.428571 & 11.428571 & 11.428571 & $\begin{array}{c}8> \\
80\end{array}$ \\
\hline
\end{tabular}

Table 2. Frequency Calculation

\begin{tabular}{|c|c|c|c|c|c|}
\hline Frame & $\mathbf{f}_{\mathbf{o}}$ & $\mathbf{f}_{\mathbf{e}}$ & $\mathbf{f}_{\mathbf{o}}-\mathbf{f}_{\mathrm{e}}$ & $\left(\mathbf{f}_{\mathbf{o}}-\mathbf{f}_{\mathbf{e}}\right)^{2}$ & $\begin{array}{c}\left(\mathbf{f}_{\mathbf{o}}-\mathbf{f}_{\mathbf{e}}\right)^{2} \\
\mathbf{f}_{\mathbf{e}}\end{array}$ \\
\hline A & 12 & 11.428571 & $\begin{array}{r}- \\
0.5714286\end{array}$ & 0.3265306 & 0.0285714 \\
\hline B & 16 & 11.428571 & 4.5714286 & 20.897959 & 1.8285715 \\
\hline $\mathrm{C}$ & 15 & 11.428571 & $\begin{array}{r}- \\
3.5714286 \\
\end{array}$ & 12.755102 & 1.1160714 \\
\hline $\mathrm{D}$ & 9 & 11.428571 & 2.4285714 & 5.897959 & 0.5160714 \\
\hline E & 16 & 11.428571 & $\begin{array}{r}- \\
4.5714286 \\
\end{array}$ & 20.897959 & 1.8285715 \\
\hline $\mathrm{F}$ & 9 & 11.428571 & 2.4285714 & 5.897959 & 0.5160714 \\
\hline G & 3 & 11.428571 & 8.4285714 & 71.040816 & 6.2160714 \\
\hline & & & & & $\begin{array}{l}\chi^{2} \\
=12.05000003\end{array}$ \\
\hline
\end{tabular}

Table 3. Descriptive Statements

$\mathrm{f}_{\mathrm{o}}$ is an experimental or experimental frequency of a video frame.

$\mathrm{f}_{\mathrm{e}}$ is an predictable frequency of a video frame. It should be $\geq 5$ when using the permanent chi-square allocation for a discrete problem.

Equal acceptance means $\mathrm{f}_{\mathrm{e}}=80 / 7=11.4285714$

$\mathrm{k}$ signifies the number of video frames.

Here k-1 degrees of freedom for a decency of fit difficulty.

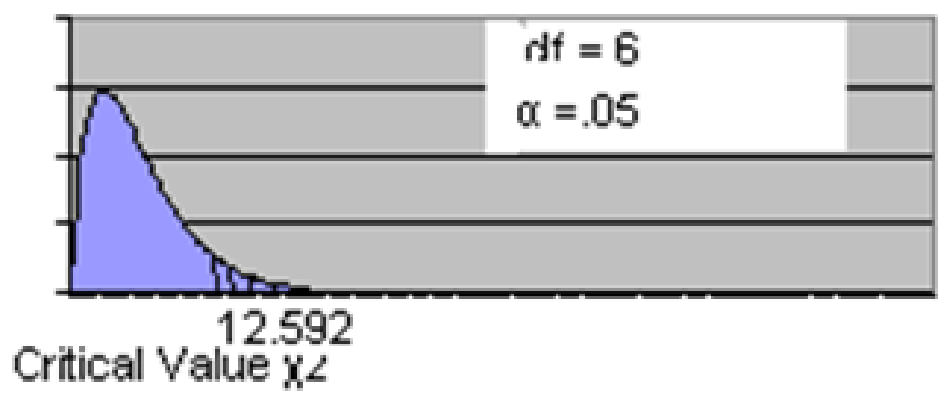

Figure 3. Critical Value

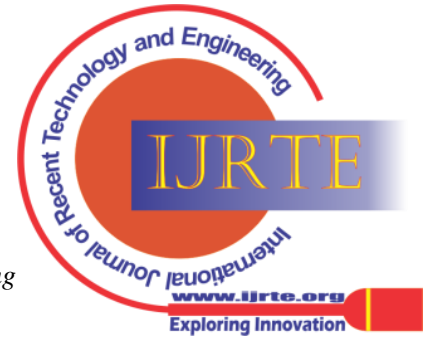


Table 5-2

Critical Values of the $\boldsymbol{x}^{2}$ Distribution

\begin{tabular}{|c|c|c|c|c|c|c|c|c|c|c|}
\hline$d f$ & 0.995 & 0.975 & 0.9 & 0.5 & 0.1 & 0.05 & 0.025 & Q.01 & 0.005 & $d f$ \\
\hline 1 & בם0 & ם0ם. & 0.016 & 0.455 & 2.706 & 3.841 & 5.024 & 6.635 & 7.879 & 1 \\
\hline$z$ & 口.010 & ㅁ.151 & $\square .211$ & 1.386 & 4.6口5 & 5.991 & 7.378 & 9.210 & 10.597 & 2 \\
\hline 3 & 0.072 & 0.216 & 0.584 & 2.366 & 6.251 & 7.815 & 9.348 & 11.345 & 12.838 & $\mathbf{3}$ \\
\hline 4 & 0.207 & 0.484 & 1.064 & 3.357 & 7.779 & $9.48 \mathrm{~B}$ & 11.143 & 13.277 & 14.860 & 4 \\
\hline 5 & 0.412 & 0.831 & 1.610 & 4.351 & 9.236 & 11.070 & 12.832 & 15.086 & 16.750 & 5 \\
\hline 6 & 0.676 & 1.237 & 2.204 & 5.348 & 10.645 & 12.592 & 14.449 & 16.812 & 18.548 & 6 \\
\hline 7 & 0.989 & 1.690 & 2.833 & 6.346 & 12.017 & 14.067 & 16.013 & 18.475 & 20.278 & 7 \\
\hline 8 & 1.344 & 2.180 & 3.490 & 7.344 & 13.362 & 15.507 & 17.535 & 20.090 & 21.955 & 8 \\
\hline 9 & 1.735 & 2.700 & 4.168 & 8.343 & 14.684 & 16.919 & 19.023 & 21.666 & 23.589 & 9 \\
\hline 10 & 2.156 & 3.247 & 4.865 & 9.342 & 15.987 & 18.307 & 20.483 & 23.209 & 25.188 & 10 \\
\hline 11 & 2.603 & 3.816 & 5.578 & 10.341 & 17.275 & 19.675 & 21.920 & 24.725 & 26.757 & 11 \\
\hline 12 & 3.074 & 4.404 & 6.304 & 11.340 & 18.549 & 21.026 & 23.337 & 26.217 & 28.300 & 12 \\
\hline 13 & 3.565 & 5.009 & 7.042 & 12.340 & 19.812 & 22.362 & 24.736 & 27.688 & 29.819 & 13 \\
\hline 14 & 4.075 & 5.629 & 7.790 & 13.339 & 21.064 & 23.685 & 26.119 & 29.141 & 31.319 & 14 \\
\hline 15 & 4.601 & 6.262 & 8.547 & 14.339 & 22.307 & 24.996 & 27.488 & 30.578 & 32.801 & 15 \\
\hline
\end{tabular}

\section{CONCLUSION}

The $(\chi 2)$ technique is a rigorous, methodical, and nonarbitrary method for determining if video data differs considerably from a previously compiled frameset and the degree of that change. The proposed model, similar to the majority statistical models, is most helpful when vast volumes of data must be analyzed and summarized for significant choices to be made.

\section{ACKNOWLEDGEMENT}

When there is dispute or lack of consensus on the importance of results, the sort of psychotherapy described at this time can be practical to the video as a whole or to precise vulnerability data. It would be simple and straightforward to implement the $(\chi 2)$ function in a software application such as Excel or SPSS to swiftly evaluate and summarize all video data. Video Magnifier [6] was the first research tool for video summarizing, and it produced summaries from a uniformly sampled video stream at specific time intervals as previously said, it would appear to be valuable from a managerial point of view to code (maybe by cluster) the squares in the video table that indicate significant values at the 0.01 and 0.05 levels and utilize this in sequence to determine which information consideration deserve follow-up. To show the direction of the change, arrows may be placed to the squares. For clustering, we investigate the use of an efficient data frame.

\section{FUTURE SCOPE}

The field of video data mining is still in its early stages. Before it becomes common practice, there are still a number of scientific issues that must be addressed. Data mining tasks in this domain encompass similarity-based processing, compression, indexing, and retrieval, information exaction, redundancy reduction, frequent pattern finding, classification, clustering, and trend and outlier detection.

Without the amazing assistance of my supervisor, Late. Rakesh Kumar Thakur, this article and the research behind it would not have been feasible. Since our first meeting, his obsession, expertise, and meticulous concentration to aspect have been an encouragement and have kept my work on pathway.

\section{REFERENCE}

1. Girgensohn, J. Boreczky, "Time-Constrained Key frame Selection Technique", 2000

2. N.D. Gagunashvili, "Chi-square tests for comparing weighted histograms", 2009

3. R. J .Perla, J . Carifio," Use of the Chi-square Test to Determine Significant of Cumulative Antibiogram Data", 2005

4. P. Turaga, A. Veeraraghavan and R.. Chellappa. "Unsupervised View and Rate Invariant clustering of Video Sequences”, 2007

5. B.H Munro, "Statistical Methods for Health Care Research". Lippincott, Williams \& Wilkins, Philadelphia, 2001

6. M. Mills, "A Magnifier Tool for Video Data", Proc. Of ACM Human Computer Interface, pp. 93-98, May 1992

7. R. Lienhart S. Pfeiffer, W. Effelsberg, "Video Abstracting", Communication of the ACM, vol.40, pp.1531-1541,1997

8. M. Smith, T. Kanade, "Video Skimming and Characterization Through the Combination of Image an Language Understanding Techniques", Proc. Of CVPR, pp. 775-781, 1997

9. R Tripathi, S. Dwivedi. A Quick Review of Data Stream Mining Algorithms. IJIR, Vol-2, Issue-7, 2016

10. R. Tripathi \& S. Dwivedi, "Accurate Career Trends Extraction for Information Professionals using Agile Text Mining", IJARCSSE, Volume 5, Issue 12, December 2015

11. R Tripathi, S. Dwivedi. Resolution Of E-Commerce Market Trend Using Text Mining. IJSRCSE,Vol.8, Issue.1, pp.01-05, February (2020)

\section{AUTHORS PROFILE}

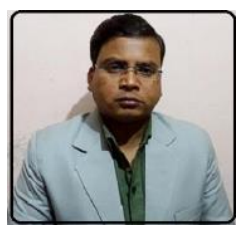

Dr. Rajeev Tripathi, Assistant Professor, Doctorate in Computer Science, MCA first division with Honours and M.Phil. in Computer Science specialization. He completed Ph.D. in 2019 in the field of Text Mining. In his Teaching-Learning journey, he also acquired M. Tech. (IT) Degree. He started his journey of academics after completing MCA in the year 2006. He is working as Assistant Professor with the Computer Application Department, Shri Ramswaroop Memorial Group of Professional Colleges, Uttar Pradesh, India. His research interests include Data Mining, Text Mining, Agile, Data Science, Machine Learning, Internet of Things and Search Optimization etc.

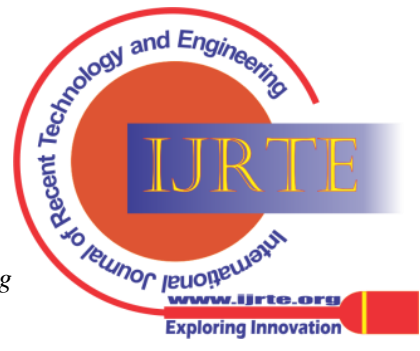

